\title{
Color vision in the comb frequency domain
}

\author{
VALÉRIE BONNARDEL* and FRANCISCO J. VARELA* \\ *Department of Psychology, University of Sunderland; Sunderland, UK \\ ${ }^{\#}$ Laboratory of Cognitive Neurosciences and Brain Imaging; Paris, France
}

\begin{abstract}
In 1982, Horace Barlow considered the question of human trichromacy in the context of information theory: according to the Sampling Theorem, three types of receptors covering the visible spectrum $(400--700 \mathrm{~nm})$ might be sufficient to reconstruct the color signal. Although Barlow was led to reject the direct application of the Sampling Theorem to explain color dimensionality, the theoretical framework offers a fresh point of view for analyzing the color system in conjunction with the physical characteristics of natural color signals. This review aims to illustrate that if the strict mathematical reconstruction (as implied by the Sampling Theorem) is replaced by a pragmatic approximation of color signals, then trichromacy, with its subsequent opponent-color process, could be regarded as an optimization of color constancy abilities in the spectral environment of primates. Higher dimension systems (tetrachromacy) found in other species can also serve the purpose of color constancy optimization in environments where color signals exhibit a finer spectral structure.
\end{abstract}

Key terms: Color vision, color dimensionality, comb-filtered spectra, color constancy, Fourier analysis.

\section{COMB-FILTERED SPECTRA}

Isaac Newton experimented with prisms breaking down the composite light of the sun into its monochromatic components circa 1666. In one experiment he placed a comb behind the prism so that regular spectral intervals were intercepted while others were transmitted. After recombining the colored beams by means of a lens, Newton could use a white sheet of paper to observe a small circle of light formed at the focal distance (Fig. 1A). The color of the circle changed successively from red to violet as the comb was slid slowly along a parallel axis. When the comb was moving faster, colors were not seen individually, and the sensation of white arose instead. This observation demonstrated yet again the fact that the sensation of white results from the mixture of all primary colors, as Newton concluded:

"... the Impression therefore of all the successive Colors are at once in the Sensorium...the commix'd Impressions of all the Colors do stir up and beget a Sensation of white, that is, that Whiteness is compounded of all the Colors" (from Newton's text Opticks published in 1730:141).

In 1982, Horace Barlow rekindled our interest in the use of comb-filtered spectra in the study of human color vision, considering them in strict analogy with the spatial frequency gratings used in spatial vision to measure the spatial resolution of the visual system. In this approach, visual stimuli are characterized by extended sinusoidal modulations in space, and defined by their frequency, amplitude, phase and mean level of energy (Fig. 1B). One can then determine the smallest spectral intervals between two consecutive maxima of amplitude or teeth of the comb that can be resolved by the chromatic system. In Newton's experiment, if the teeth of the comb covered $100 \mathrm{~nm}$ and were spaced out by an interval of the same size (i.e. equivalent to $1 \mathrm{c} / \mathrm{T}$, where $\mathrm{T}$ is the extent of the visible spectrum 400-700 $\mathrm{nm}$ ), the change of color produced when the comb slid over the spectrum (equivalent to a change of phase, Figure 2, top panel) would have been dramatic. With a reduction in 


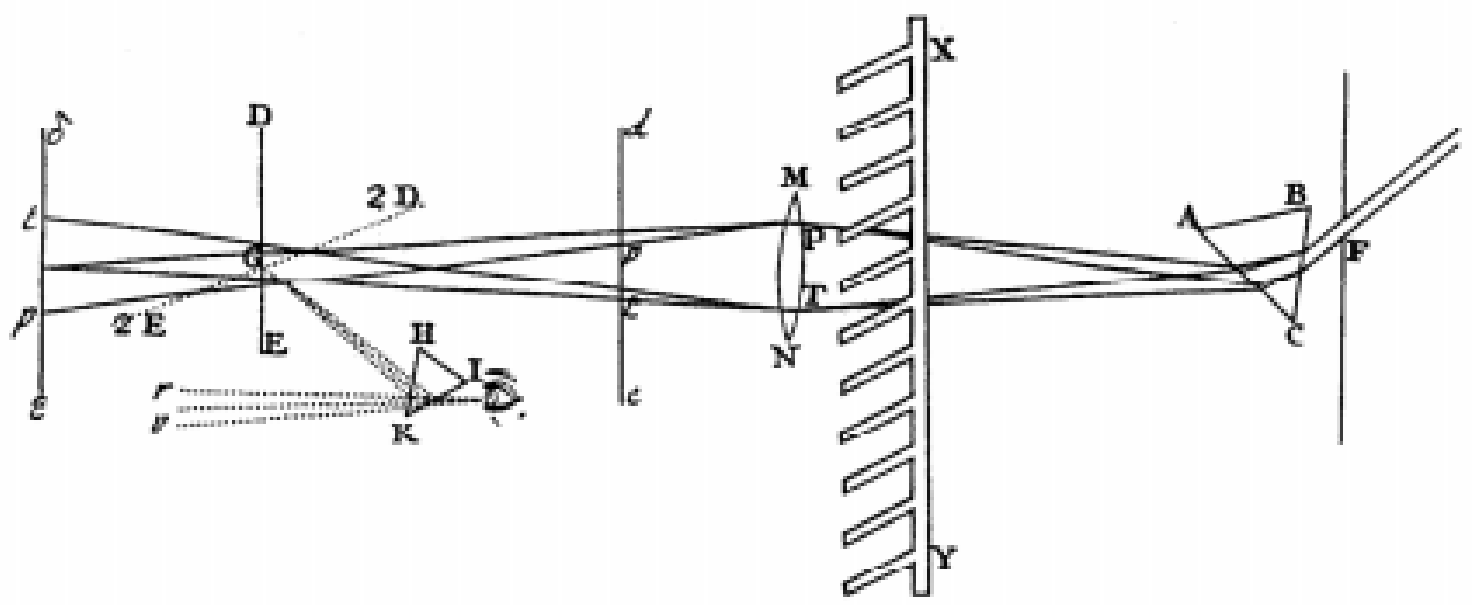

Fio, 6.

\section{Figure 1}

A. The sunlight is transmitted through a little hole in the window shutter $(\mathrm{F})$ and then refracted by a prism $(\mathrm{ABC})$ that casts its image $(\mathrm{PT})$ on a lens $(\mathrm{MN})$. At the focal distance of the lens $(\mathrm{G})$, the refracted beams are recombined and form a little white circle on a white sheet of paper (DE). When the paper is moved below $(\mathrm{de})$ or beyond $((\delta \varepsilon))$ the focal point, the colored image of the spectrum appears in the original $(\mathrm{pt})$ or reverse order $(p t)$. If a comb (XY) is placed between the prism and the lens, by intercepting certain spectral intervals and transmitting others, the little circle changes from white to a certain color that is determined by the mixture of the transmitted intervals.

the size of teeth and intervals, equivalent to $3 \mathrm{c} / \mathrm{T}$ for example (Figure 2, middle panel), the change in color would be more subtle or even non-perceptible for higher comb frequencies. This is due to the fact that the spectral power distribution (energy per wavelength) of the light is not measured by an array of sensors; rather the continuous spectrum is encoded by the photon absorption rates in a limited number of mechanisms (three for a trichromatic system) that are maximally responsive at different locations in the spectrum (Figure 2 , bottom panel). Considering the large absorption curves of the photoreceptors, the photon absorption rate in each type of photoreceptors for a comb-filtered spectrum of high frequency is similar at all phases; a shift of phase is barely detected, and since each type of photoreceptors is similarly stimulated, the comb-filtered spectrum is indistinguishable from a continuous spectrum. The comb frequency at which this confusion occurs is taken as the spectral resolution or frequency cut-off of the color system.

\section{Band Limitation and the Sampling Theorem}

Barlow's original analysis relied on estimating the modulation transfer function (MTF) of color mechanisms, which indicates the level of demodulation imposed on comb-filtered spectra of different frequencies. Due to their broad spectral sensitivity curves, human photoreceptors act as low-pass filters, as can be seen from their response in the comb frequency domain where frequencies greater than $6 \mathrm{c}$ / T are filtered out (Fig. 3). After receptor filtering, the effective signal is band or frequency limited (low frequency components are also demodulated) and can be reconstructed by a finite number of samples (i.e. type of photoreceptors) within a defined spectral interval (i.e. the visible spectrum). The number (N) of samples can be deduced from the application of the Sampling Theorem, which states that to recover a signal of extent $T$ (i.e. wavelength domain) and of frequency cut-off $\mathrm{B}$ (i.e. comb-frequency domain), $\mathrm{N}=2 \mathrm{BT}$ (see Appendix A). Such a relationship could offer an explanation for human trichromacy. 


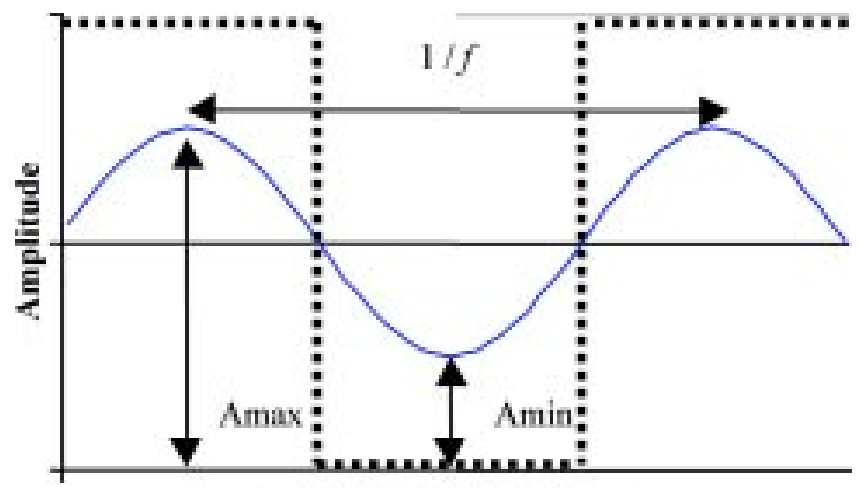

\section{Figure 1 - (B and C)}

Time, space or wavelength

B. The interest in using sine wave functions as a stimulus is obvious in the sound domain where sound vibrations of any complexity can be broken down into sine and cosine waves of specified frequencies, amplitudes and phases, corresponding to pure tones. This operation is known as representing the stimulus in the frequency domain, and in practice it is carried out by the Fourier transform or Fourier analysis. The frequency domain approach was later successfully introduced in the spatial domain, in which complex visual patterns can be constructed as sinusoidal modulations of luminous energy. The response and receptive field of the visual mechanisms can thus be characterized precisely. In the spectral domain, sinusoidal modulations of the spectral energy are defined as:

$$
\mathrm{E}(\lambda)=\mathrm{E}_{0}\left[1+\mathrm{A} \sin \left(f p(\lambda)+p_{0}\right)\right]
$$

with $E_{0}$ : the mean energy level; $f$ : the comb frequency expressed in cycles / visible window $\mathrm{T}$ which corresponds to $300 \mathrm{~nm}$ for human vision (from 400 to $700 \mathrm{~nm}$ ), hereinafter noted c / T; A : the amplitude of the modulation; and $p_{0}$ : the phase (in degrees). $p(\lambda)=1.2 \lambda-480$ is used to scale the $400-700$ interval into $360^{\circ}$.

Strictly speaking, Newton's comb would produce square (dotted line) or rectangular modulations of the spectral energy; however, the term 'comb-filtered spectra' refers here to periodical modulation of the spectral energy.

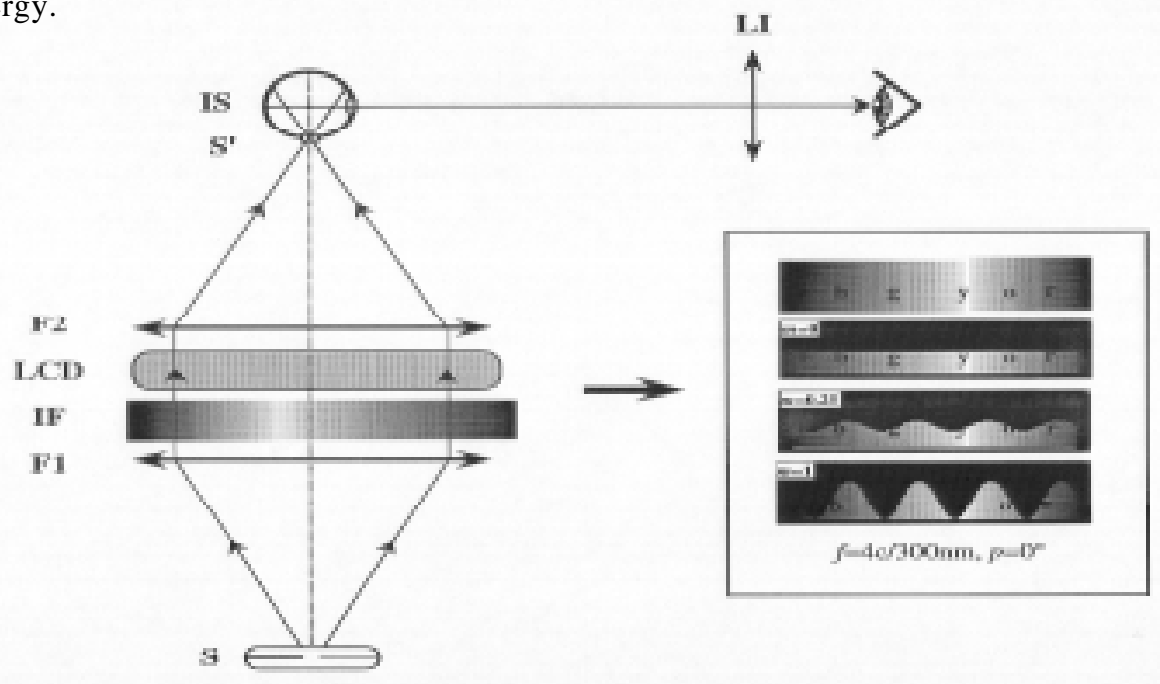

C. The light from the source (S) is located at the focal point of a Fresnel lens (F1), and focused by a second lens (F2) to form a real image S' of S at the aperture of the integrating sphere (IS). The LCD is mounted in the collimated beam directly after the interference wedge (IF) which gives a continuous linear spectrum from 400 to $700 \mathrm{~nm}$. The output of the integrating sphere, viewed through a lens (L1), produces a homogeneous spot. The figure on the right shows examples of comb-filtered spectra achieved by applying electronic masks to the spectrum (symbolized by the color initials). The masks presented here have $f=4 \mathrm{c} / \mathrm{T}$ and $p=0 \mathrm{deg}$ with 3 different amplitudes. When $m: 0$ the spot appears colored, and for constant amplitude its hue depends on the combination of frequency and phase, that is, the number and position of the spectral bands transmitted. 

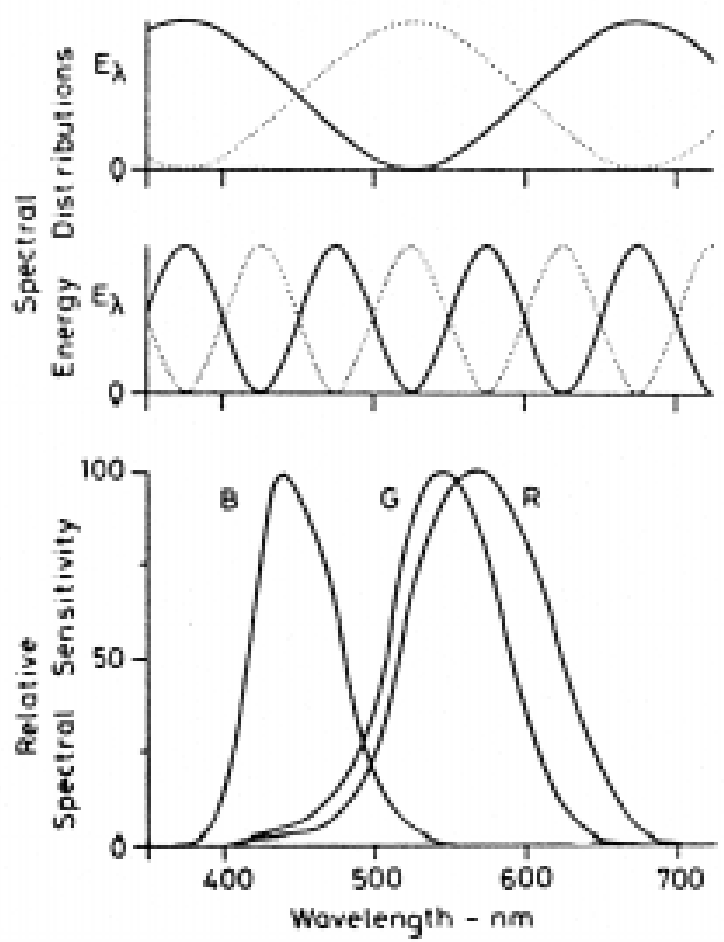

Figure 2

The top two sections show comb-filtered spectra of 1 and $3 \mathrm{c} / \mathrm{T}$, with two phases $180^{\circ}$ apart. The lowest section shows the three cone-fundamentals (B G R) of Smith and Pokorny (1975) plotted in relative units. The cone-fundamentals represent the sensitivity of the photoreceptors at the corneal level. The color of the $1 \mathrm{c} / \mathrm{T}$ comb-filtered spectra will change dramatically with a shift of phase (from violet to greenish-yellow) and a large demodulation of amplitude will be necessary to make this change undetectable. The change in color will be far less prominent for a comb frequency of $3 \mathrm{c} / \mathrm{T}$, even with large modulation of amplitude. (From H. Barlow, 1982).

If the number of photoreceptors satisfies the equation, adding another sampling dimension (e.g. a fourth type of photoreceptors) would be redundant. For a trichromatic system, the equation becomes $3=2 \mathrm{BT}$, yielding a maximum value of $\mathrm{B}=$ $1.5 \mathrm{c} / \mathrm{T}$ to satisfy the Sampling Theorem's strict limit. However, the actual frequency cut-off of the photoreceptors $(4-6 \mathrm{c} / \mathrm{T})$ would require 8 to 12 types of photoreceptors for the accurate reconstruction of a signal with a similar frequency limit. Barlow was thus led to reject the direct application of the Sampling Theorem as an explanation of human trichromacy and his analysis reveals the existence of signal aliasing occurring at the receptoral level, producing what is known as color metamerism.

This review aims to re-examine the combfrequency approach to color vision by considering the filtering properties of the color system in relation to the neural processing of natural color signals. Barlow's original analysis will be extended to encompass current knowledge of the characteristics of natural color signals encountered in primates' environment. Linear models of color constancy that assume signal approximation will then be introduced. This analysis must indeed be based on color constancy, as this establishes the need for the chromatic system to approximate the incoming signal; color discrimination would not require such process. We will next consider the transfer characteristics of the chromatic system beyond the receptor level. From this analysis we conclude that if an exact mathematical reconstruction of the color signal could not be achieved by a trichromatic system, a pragmatic

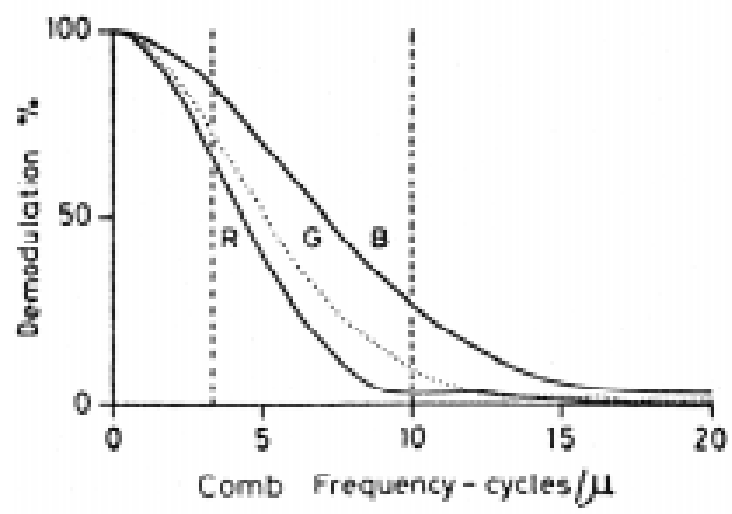

From H. Barlow, 1982

Figure 3

Fourier transform of Smith and Pokorny (1975) cone fundamentals. At $3 \mathrm{c} / \mathrm{T}$ (indicated by the leftmost vertical dotted line), the demodulation is approximately $25 \%$ for the short-wavelength (B) and approximately 12 and $4 \%$ for the medium- and long- wavelength cone fundamentals ( $G$ and $R$ ), respectively. The abscissa axis is expressed in $\mathrm{c} / \mathrm{B}$ where 1 cycle corresponds to $0.33 \mathrm{c} / \mathrm{T}$. 
approximation carried out by well-designed opponent-color channels could have taken place to secure a suitable degree of color constancy. This conclusion leads to the conjecture that tetrachromacy, in addition to its adaptation to a wider visible spectrum, can also serve the purpose of color constancy optimization in spectral environments of greater complexity than those of trichromats.

\section{Band limitation of natural color signals}

Color signals are physically constrained to be band-limited or low-pass functionsfunctions that do not have frequency components beyond a certain limit. This claim, which dates back to Stiles et al (1977), was made from the mere observation that natural color signals generally have smooth spectral profiles.

The color signal $1(\lambda)$ results from two intertwined functions: the spectral power distribution of the illuminant $\mathrm{e}(\lambda)$ and the spectral reflectance of the object surface $\mathrm{s}(\lambda)$, so that: $1(\lambda)=\mathrm{e}(\lambda) \mathrm{x} s(\lambda)$. To estimate the color signal frequency cutoff, the two components need to be considered in turn. Maloney (1986) provided the first direct estimate obtained for spectral surface reflectances from stained color samples used in industry (Nickerson-Munsell chips) and surfaces of natural formations (measured by Krinov in 1947). At least ninety-nine percent of the energy in the Fourier transform power spectra was recovered for frequencies as low as $1.5 \mathrm{c} /$ $\mathrm{T}$, and all energy was recovered for frequencies of less than $6 \mathrm{c} / \mathrm{T}$. A second analysis performed by van Hateren (1993) on the spectra of various natural objects showed an identical frequency cut-off. However, these studies could be criticized because they originate from stained samples (Nickerson-Munsell chips) and a heterogeneous collection of objects (Krinov and van Hateren's samples), and therefore the results cannot be generalized to spectral reflectance surfaces present in the natural environment or attributed to a specific category of natural colors.
More recently, Bonnardel and Maloney (2000) performed a Fourier analysis of the spectral reflectance functions arising from fruits, flowers, and leaves. These natural surfaces, described as biochrome colors (Nassau, 1983), owe their coloration to the selective absorption of incident light by pigments. This process of light reflection is commonly referred to as 'body reflection' as opposed to 'interface reflection' (also known as specular reflection) (these two processes are described in the Dichromatic Reflection Model proposed by Shafer (1985)). Body reflection is diffuse as the light is reflected in many different directions, and the spectral composition of the light reflected does not depend upon the viewing geometry but is determined by the property of the pigment particles and thus is indicative of the intrinsic color of the material. In contrast, interface reflection is concentrated in one direction, is dependent upon the viewing geometry and for many types of materials the reflection is non-selective, and can thus be indicative of the spectral properties of the illuminant. As we will see later in this paper, the explanation of the light reflection process in terms of physico-chemical properties is important for a proper characterization of the color signal.

The FFT performed on 1695 biochrome samples indicates that more than $99 \%$ of the energy is recovered for frequencies below $4 \mathrm{c} / \mathrm{T}$ (Bonnardel and Maloney, 2000). This limit is somewhat higher than that obtained in Maloney's analysis; however, only a residual amount of energy $(<0.005)$ is found at frequencies higher than $6 \mathrm{c} / \mathrm{T}$. The latter result confirms the low-pass hypothesis of the reflectance functions of biochrome surfaces. Due to the lack of availability of empirical measurements, estimates of the cutoff frequency for natural illuminants are far less numerous. However, in the same study a second FFT was performed on a small sample of 348 daylight spectral power distributions provided by Romero et al (1997). Results show that more than $99 \%$ of power density is recovered below $1 \mathrm{c} / \mathrm{T}$.

From these two estimates, we can deduce the color signal frequency cut off. The 
product of the two functions $e(\lambda) \times s(\lambda)$ when transposed into the frequency domain corresponds to a convolution of their Fourier transforms. It can be shown that the frequency limit of the product is at least as high as either the frequency limit of the illuminant $(b)$ or the surface reflectance ( $b$ '), but less than the sum of the two frequency limits $\left(b+b^{\prime}\right)$ (Bracewell, 1965). It follows that the upper limit of the frequency cut off of the color signal reflected by biochrome surfaces under daylight illuminant for the samples considered was estimated at $5 \mathrm{c} / \mathrm{T}$ for the criterion of $99 \%$.

\section{Linear models of color constancy}

Color constancy can be described as the tendency to see colors as unchanging even under changing illumination condition' and computational models are designed to recover the invariant spectral reflectance properties of object surfaces from the color signal (Hurlbert, 1998). Given that color signals are band-limited functions, as indicated by the previous analysis, linear models of color constancy offer a computational method for recovering the descriptors of the surface reflectances. Band-limited functions can be adequately approximated by a small number of basis functions whose number, as stated by the Sampling Theorem, is proportional to the frequency limit of the color signal. Thus, a set of illuminants will be approximated by a limited number of illuminant basis functions $E_{i}(\lambda)$ :

$$
e(\lambda) \approx \varepsilon_{1} E_{1}(\lambda)+\ldots+\varepsilon_{m} E_{m}(\lambda)
$$

Likewise, a set of surface reflectances will be approximated by a limited number of surface reflectance basis functions $S_{j}(\lambda)$ : $s(\lambda) \approx \sigma_{1} S_{1}(\lambda)+\ldots+\sigma_{n} S_{n}(\lambda)$

the $\varepsilon_{\mathrm{i}}$ and $\sigma_{\mathrm{i}}$ are the weights or the descriptors of the illuminant and surfaces and the quality of the approximation increases with the number $(n, m)$ of basis functions ${ }^{1}$. For the color system to solve the color constancy, the descriptors of the illuminant and surface need to be recovered. The response $\mathrm{Q}$ of $p$ types of receptors with response function $q_{k}(\lambda)$ to the color signal is :

$$
Q_{k}=\sum_{\lambda} e(\lambda) s(\lambda) q_{k}(\lambda)
$$

$k=1,2, \ldots, p$

If $e(\lambda)$ and $s(\lambda)$ are replaced by their linear models expressed in eq. [1] and [2], the receptor response becomes:

$$
Q_{k}=\sum_{i=1}^{m} \sum_{j=1}^{n} \varepsilon_{i} \sigma_{j} \sum_{\lambda} E_{i}(\lambda) S_{j}(\lambda) q_{k}(\lambda)
$$

In conditions where the illuminant can be known, i.e. the $\varepsilon_{i}$ can be recovered ${ }^{2}$ to find a solution to eq. [4], the number of unknown descriptors $\sigma_{i}$ of surface reflectance should not exceed the number of type of receptors. In practical terms, this number should not exceed 3 for a trichromatic system, hence the importance of verifying the quality of the approximation of natural reflectance surfaces with low-dimension linear models.

It is possible to derive a set of basis functions from a set of measured reflectance functions using Principal Component Analysis (PCA) and to determine which contributes the most to the total variance of that particular set. Cohen (1964) applied the PCA to 433 Munsell chips and found that $99.18 \%$ of the variance can be accounted for by the first three basis functions, although Parkkinen et al. (1989) estimated that the variance could be accounted for by as many as 8 basis

\footnotetext{
${ }^{1}$ Illuminants are probably approximated by a linear model of smaller dimension compared to that of surface reflectances. From their own daylight measurements Romero et al. (1997) confirmed Judd, MacAdam and Wyszecki (1964) earlier result that three basis functions are suitable to describe natural daylight and reported a percentage as high as 99.97 of the total variance recovered. This value is superior to any value obtained for natural surface reflectances (see text)
}

${ }^{2}$ The illuminant can be known through specular reflection for instance, but see Maloney (1999) for a detailed review of the different methods and assumptions used in computational approaches to solve this question 
functions for the complete set of Munsell chips (1257) when accuracy of the approximation is used as a criterion instead of the percentage of the total variance. Similarly, using the accuracy criteria, Vrhel et al (1994) found that 3 to 7 basis functions were necessary to describe a set of heterogeneous spectra (Munsell chips, Du Pont paint chips and natural and man-made objects). Finally, $98 \%$ of the total variance of forest and coral scenes is also recovered with the first three basis functions in a study recently done by Chiao et al. $(2000)^{3}$.
Basis functions determined for a given sample would not necessarily provide a satisfactory fit with data from another sample, but they do share the same characteristics in all the studies cited. While the first basis function is an all-positive function with a generally flat profile, the second has one zero-crossing and the third, two zero-crossings along the wavelength axis (Fig. A). The profile of the basis functions does not correspond to that of the photoreceptors, and in linear models of color constancy the receptors should first

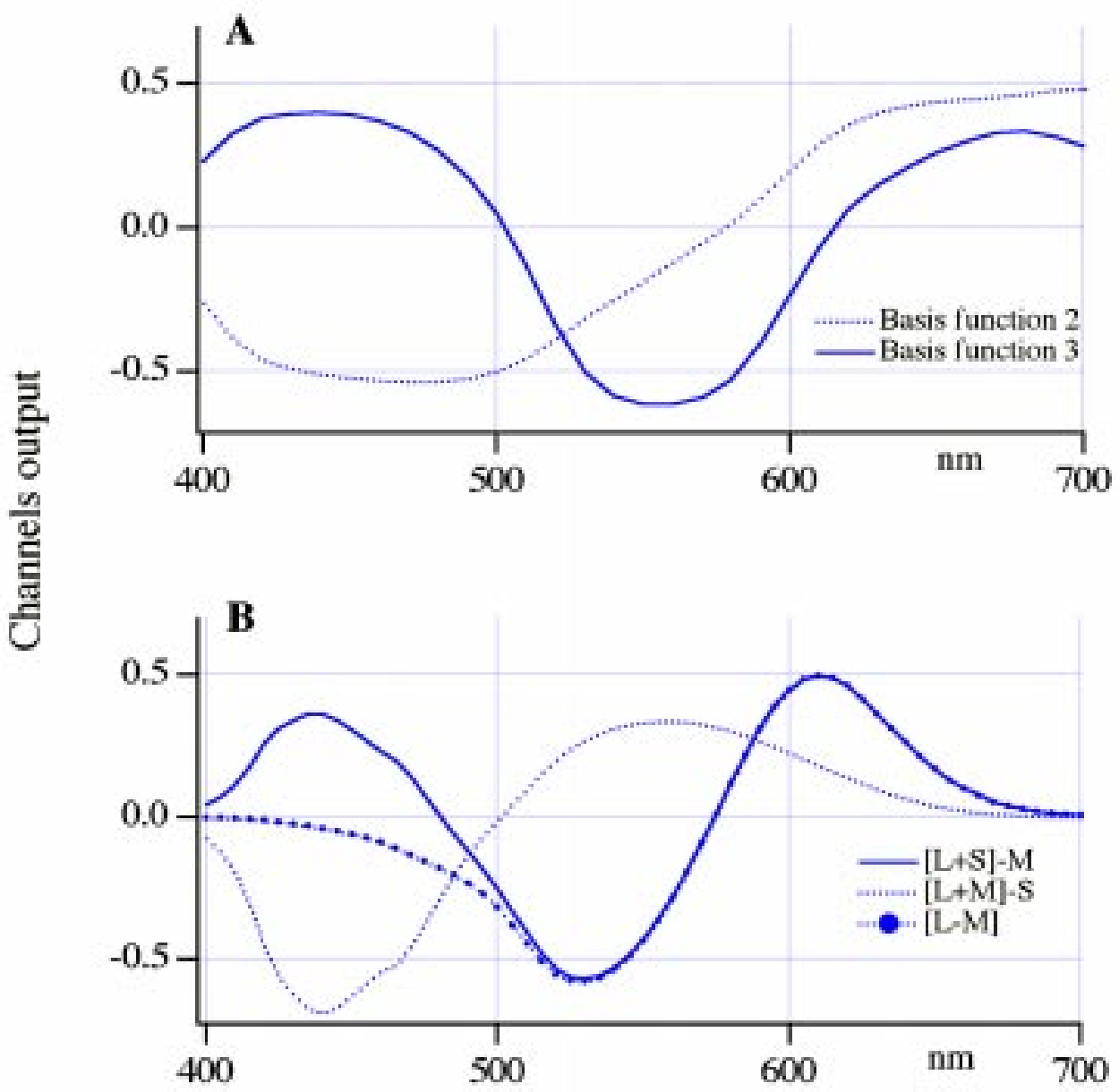

Figure 4

A: Second and third basis functions determined from the PCA of heterogeneous spectral reflectance functions by Vrhel et al. (1994).

B: Opponent-color channels resulting from the linear combination of the Smith and Pokorny (1975) fundamentals as proposed in Ingling and Tsou's model (1977). The profile of $[\mathrm{L}+\mathrm{S}]-\mathrm{M}$ and $[\mathrm{L}+\mathrm{M}]-\mathrm{S}$ is comparable to that of the basis functions.

\footnotetext{
${ }^{3}$ However, the coral spectra were smoothed out with a low-pass filter of $6 \mathrm{c} / \mathrm{T}$ (which corresponds to a sampling of $25 \mathrm{~nm}$ ) thus limiting the estimation of the percentage of variance at this frequency.
} 
undergo a linear transformation into a new set corresponding to the basis functions in order to achieve color constancy (Hurlbert, 1998). This new set of functions is actually found in the visual system and corresponds to the opponent color stage that follows receptor response. The two post-receptoral opponent color channels also exhibit one or two zero-crossings somehow shifted towards the short wavelengths, compared to the computed basis functions (Figure 4B). Although not identical to the basis functions they are comparable.

\section{Filtering properties of the human color system}

The two stages of color processing mentioned above were the subject of the conflict between Young's trichromatic theory (1802), which proposed the existence of three distinct types of receptors, and Hering's opponent color theory (1878), which was developed to account for sensation phenomena left unexplained by the former theory. The controversy was later resolved by integrating the two processes in multi-stage models of color vision (e.g. Hurvich and Jameson, 1957).

The two opponent-color channels result from a linear combination of the three types of photoreceptors: A 'yellow-blue' channel receives combined inputs from the mediumand long-wavelength receptors (M-and Lcones) in opposition to inputs from the shortwavelength receptors (S-cones) and exhibits a bimodal spectral profile $[\mathrm{L}+\mathrm{M}]-\mathrm{S}$. A 'red-green' channel combines L-cones in opposition to M-cone inputs [L - M], which also produces a bimodal spectral profile. Under certain conditions, this second channel may receive $\mathrm{S}$-cone inputs leading to a 'cyan-red' (S-cones combined to $\mathrm{M}$ cones) or a 'magenta-green' opponency (Scones combined to L-cones). Although cells in the lateral geniculate nucleus (LGN) of primates respond preferentially to yellowblue and red-green modulations (Derrington et al. 1984), cells responding to cyan-red or magenta-green modulations might be found in the cortex and were recently described in area V1 of the primate cortex (Conway,
2000). In Ingling and Tsou's model (1977), the two versions of the red-green channel are expressed as a linear combination of two $[L$ - M] or three $[L+S]-M$ type of photoreceptors, depending on the light adaptation conditions. The two channels show a di- or triphasic spectral profile, respectively (Figure $4 \mathrm{~B}$ ).

Due to their opponent process, the postreceptoral channels show band-pass filter characteristics in the comb frequency domain, with a high frequency cutoff equivalent to that imposed by the receptors. Moreover, they attenuate low-frequency components. Depending on the type of cone interactions, the channels are tuned to different ranges of comb frequencies $([\mathrm{L}+$ $\mathrm{M}]-\mathrm{S}$ channel peaks at $1 \mathrm{c}$; [L - M] channel at $1.2 \mathrm{c}$ and the $[\mathrm{L}+\mathrm{S}]-\mathrm{M}$ channel at 1.5 c / T). The yellow-blue channel is thus involved in the extraction of lower comb frequencies compared to the red-green channel, but the difference in tuning is even more marked when compared to the magenta-green channel, which shows a further shift to higher comb-frequencies in its response (Fig. 5a) In the absence of one type of retinal receptor, as is the case in inherited human dichromacy (Nathans et al. 1986) the best that can be achieved is a single channel with a simple cone opponency $[\mathrm{L}-\mathrm{S}]$, for deuteranopy or [M $\mathrm{S}$ ] for protanopy. The net consequence is a reduction in the width of the tuning curve in the comb frequency domain (Figure 5B).

Measurements of human sensitivity to comb-filtered spectra were performed for the first time by Barlow et al (1983) using a Michelson interferometer. Sensitivity thresholds plotted as a function of comb frequencies exhibit curves with jagged profiles, indicating the high degree of dependency of the color system on phase (i.e. in a Michelson interferometer the phase of the comb-filtered spectra varies with the comb frequency). In 1991, we developed a stimulator that allows us independent control of the modulation parameters (Bonnardel and Varela, 1991). In its latest version (Bonnardel, 1998), the stimulator uses the same optical arrangement as in Newton's set-up. The main difference lies in the use of a linear interferential filter to 

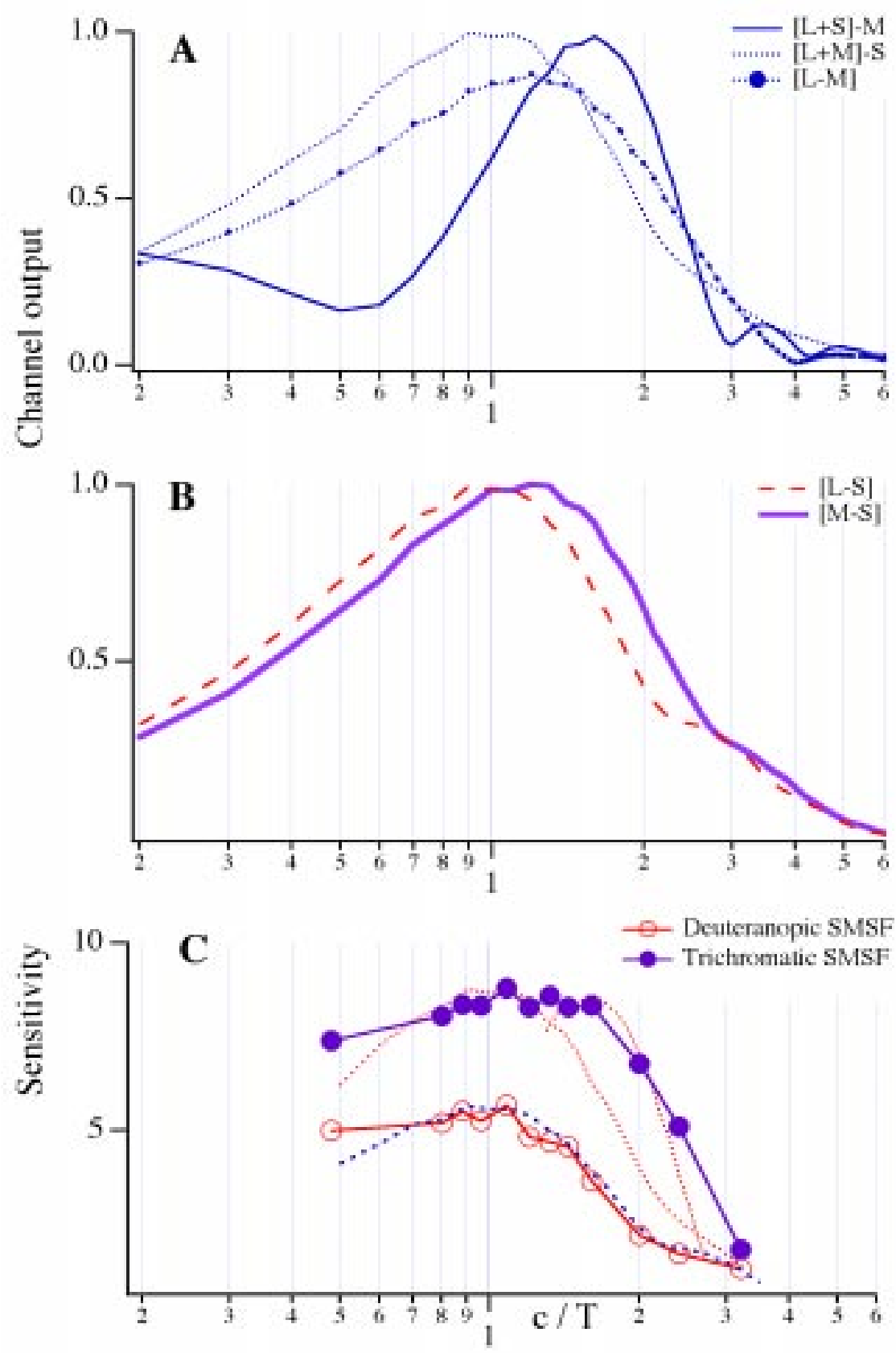

Figure 5

Response of the human chromatic system to comb frequencies at various levels of description:

A. Normalized response of opponent-color channels [L+M] - S, [L-M] and [L+S] - M of Ingling and Tsou's model $(1977)^{4}$;

B. Normalized response of opponent-color channels derived for a deuteranopic [S-L] and a protanopic [SM] system by the simple suppression of the missing cone fundamental in Ingling and Tsou's model (1977).

C. Mean spectral modulation sensitivity function of normal trichromat and deuteranopic observers adapted from Bonnardel et al. (1997).

${ }^{4}$ The response of cone fundamentals and opponent-color channels are computed for comb frequencies varying from 0.1 to $6 \mathrm{c} /$ $\mathrm{T}$ (in intervals of $0.1 \mathrm{c} / \mathrm{T}$ ) and for phase, varying from 0 to $330^{\circ}$ (in intervals of $30^{\circ}$ ). For each phase the value for the optimal phase only is plotted 
obtain a linear spectrum, and the use of a Liquid Crystal Display to replace the comb, thus providing the advantage of displaying electronic masks of any desired spectral profile under computer program control (Figure 1C). The type of measurements obtained were defined as the Spectral Modulation Sensitivity Function (SMSF) (Bonnardel et al. 1996), which corresponds to sensitivity to the optimal phase plotted only as a function of the comb frequency. The SMSF thus provides an estimate of the modulation transfer characteristics of the chromatic system. Several studies have since followed (Bonnardel et al. 1997; Romero et al. 1997) in which, despite differences in technique and methods, all SMSFs show band-pass profile with a maximum sensitivity between 1 and $1.6 \mathrm{c} /$ $\mathrm{T}$ and a high comb frequency cutoff estimated between 4.5-6 c/ T.

In Figure 5C, the SMSF determined by trichromatic observers is well fitted in the low comb frequencies by the response function of the yellow-blue channel and in the high comb frequencies by the magenta - green (triphasic) channel. However, the red-green (diphasic) channel fails to fit the second shoulder situated at $1.5 \mathrm{c} / \mathrm{T}$. The SMSF of deuteranope observers shows a corner frequency of $1.1 \mathrm{c} / \mathrm{T}$ with a reduction in sensitivity in the comb frequency domain which is consistent with the lack of redgreen opponency. The curve's profile is reasonably well described by the response of the red-blue channel.

\section{Color dimensionality}

The analysis of biochrome colors and the filtering properties of the color system allow us to draw specific conclusions regarding the relationship between color dimensionality and color constancy for human color vision. A more general conclusion with respect to coherent bandpass theory that applies to color vision in other species is proposed.

1- The frequency cut-off of biochrome colors for the criterion of $99 \%$ coincides with the frequency cut-off of the photoreceptors. This coincidence might not be fortuitous. The molecularinteraction hypothesis, as proposed by Maloney (1986), offers an explanation of the observed low-pass constraints based on the physics and chemistry occurring at the molecular level for biochrome colors, where colors are produced by selective absorption of light by pigments. This explanation should also apply to visual pigments in which the combination of a protein (opsin) with an aldehyde of vitamin A (retinal) should yield a similar cut-off frequency.

2- As already pointed out by Maloney (1986), the number of types of receptors is not dictated by the signal approximation requirement; if it were, a perfect color constancy would be expected, and this is obviously not the case for human color vision. However, although the system largely undersamples the signals, Maloney (1986) showed that the low pass filtering properties of photoreceptors enhance their approximation. At the second stage of color processing, in his spatio-temporospectral optimal filter theory, van Hateren (1993) demonstrated that, by attenuating the lower frequencies, opponent-color mechanisms exhibit an elementary form of color constancy. This filtering process primarily concerns illuminants that, as mentioned earlier, correspond to the lowest comb frequency components in the color signals. The windowing imposed at the color opponent stage can thus be regarded as the second step of signal filtering to optimize the signal approximation that can be performed at later stages.

3- Buchsbaum and Gottschalk (1983) showed that the design of the two opponentcolor channels corresponds to the optimum transformation from the three overlapping types of receptors, to an optimization of information transmission by decorelating receptor output and thus reducing redundancy. As discussed previously, Buchsbaum and Gottschalk's opponentcolor functions shared the same characteristics as the basis functions, which, as noted by Hurlbert (1998) is nonaccidental: both analyses undergo a 
decomposition into principal components. However, the opponent-color channels postulated by a psychophysical model such as Ingling and Tsou (1977) bear a closer resemblance to the functions resulting from Buchsbaum and Gottschalk's optimum transformation than to basis functions. In the comb frequency domain, the opponentcolor channels outperform the basis functions, extending sensitivity to high frequencies (Figure 6). This result can be attributed to the fact that the basis functions so far computed are derived from a limited set of reflectances and, apart from the study of Chiao et al. (2000), computations made by excluding the contribution of the illuminant did not consider the color signal per se.

If color constancy performance is dependent upon the number of receptors and the subsequent transformation of their response, this raises the question of maintaining color constancy in the case of innate dichromats, whose color system is missing the red-green opponent mechanism. Although this mechanism has a crucial ecological role in the detection and discrimination of fruits (Regan et al. 1998) or young leaves (Dominy and Lucas, 2001) against mature foliage (and is indeed believed to have evolved to fulfill this function in frugivore and folivorous monkeys), the performance of daltonians

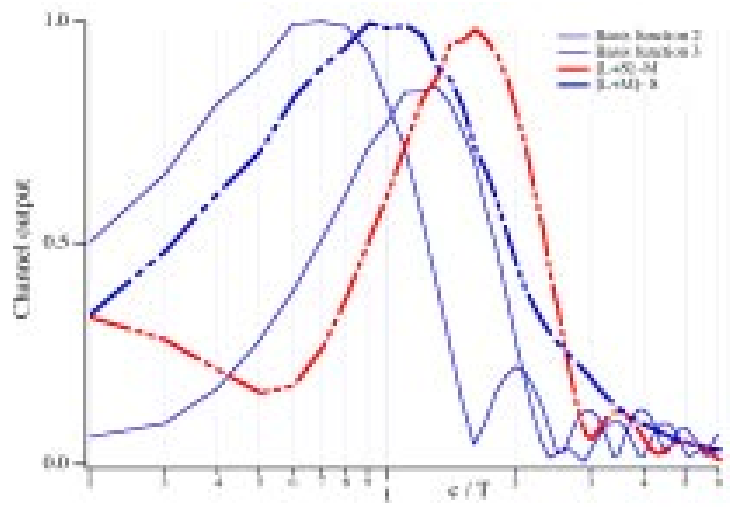

\section{Figure 6}

Normalized response in the comb-frequency domain of the second and third basis functions (continuous line) presented in figure 4A compared to that of the opponent-color mechanisms (dashed line) presented in figure $4 \mathrm{~B}$. does not seem to be very impaired when tested for color constancy under laboratory conditions (Ruttiger et al. 2001). The lack of obvious color constancy impairment could be partially explained by the more drastic filtering process imposed by the yellow-blue mechanism, which helps to retrieve color descriptors with only two basis functions. However, if dichromats' failure in color constancy seems less obvious than their color confusions under laboratory testing conditions, observed results are different in natural settings. It is from a lack of constancy in the color appearance of a pelargonium when moving from candlelight to daylight that John Dalton gained his first acquaintance with his visual condition (Collins, 1925). Indeed, color signals under candlelight compared to daylight illuminants might have a slightly smaller percentage of the total variance recovered at $1 \mathrm{c} / \mathrm{T}$ (limit of recovery for a 2-dimensional linear model). In addition, more basis functions would be required to recover surface descriptors seen under this illuminant (see Table I, for the percentage of total energy recovered for a biochrome color under a tungsten bulb and daylight illuminant). In the natural environment, certain conditions might be more challenging to dichromats, thus revealing the more obvious failure of color constancy. It would complement our argument if, in relation to the geographical distribution of human color deficiencies as given in Cruz-Coke's (1964) survey, specific changes in light and/or surface were found to be more critical for color deficient color constancy.

In light of what has been presented, we can now reconsider the question behind Barlow's interest in comb-filtered spectra: Why trichromacy? It is clear that there are a number of factors that must enter into an understanding of color dimensionality: (1) the band limitation in natural color signals; (2) the frequency characteristics of photoreceptors; (3) the constraints to achieving color constancy beginning with color signals and photoreceptor responses and the establishment of color-opponent stages. These different factors are, to varying degrees, relatively independent 


\section{TABLE I}

At $1 \mathrm{c} / \mathrm{T}$ the percentage of total energy recovered from an orange is lower under the tungsten bulb than under daylight illuminant. In this example, the structural color signal displays

higher cut-off frequencies than the biochrome color signal (shaded cells) (see text for explanation).

\begin{tabular}{|c|c|c|c|c|c|c|}
\hline \multicolumn{7}{|c|}{$\begin{array}{c}\text { Proportion of spectral energy below the frequency-limit (c / T) } \\
\text { Visible window : 400-710 nm }\end{array}$} \\
\hline & 1 & 2 & 3 & 4 & 5 & 6 \\
\hline Orange $\mathrm{x}$ Daylight & 0,8845 & 0,9958 & 0,9979 & 0,9983 & 0,9985 & 0,9986 \\
\hline Orange $x$ Tungsten & 0,8572 & 0,9962 & 0,9988 & 0,9995 & 0,9995 & 0,9996 \\
\hline Blue feather x Daylight & 0,7562 & 0,9505 & 0,9910 & 0,9967 & 0,9975 & 0,9981 \\
\hline Blue feather $\mathrm{x}$ Tungsten & 0,8455 & 0,9583 & 0,9882 & 0,9957 & 0,9972 & 0,9981 \\
\hline
\end{tabular}

from one another, and include: sources of illumination, structure of surface materials, receptor photochemistry derived from ancestral biochemical pathways (Nathans et al. 1986), neural developments, and the behavioral and ecological requirements of color constancy (Thompson et al. 1992; Thompson, 1995). The result achieved through the biological evolution of all of these relatively independent natural factors can be assumed to originate from a coherent relationship. No single aspect involved in color vision can in itself account for trichromacy; it is the mutual constraints among them that lead to an evolutionarily satisfactory color dimensionality in the primate group. In other words, there are plenty of metamerisms with 3 types of receptors (and even more with two), but the signal windowing achieved at the postreceptoral level allows a 'good-enough' signal approximation for the purpose of color constancy with three or two basis functions. This 'sufficing' argument provides a well-grounded rationale for the fact that humans are trichromats rather than deca-chromats, if we follow the strict limit of the Sampling Theorem. Trichromacy appears to be an ecological compromise between accuracy and simplicity in the use of resources; we may call this multi-factor account of trichromacy in primates a coherent band-pass theory of color dimensionality.
Implications for the color systems of other species

Considering the central role of color constancy in object recognition, it is a reasonable conjecture that this property has been subject to a selective pressure that presumably makes it inseparable from the evolution of color vision itself in the entire animal kingdom (Thompson et al., 1992). Indeed, in addition to primates, insects, fishes and birds exhibit color constancy when tested behaviorally (Neumeyer, 1998). The coherent band-pass theory of color dimensionality can thus be applied to tetrachromatic systems.

Tetrachromacy is a common feature among insects, fishes, reptiles and birds (Goldsmith, 1991). In these species, the addition of a fourth type of receptors in the context of the comb-frequency analysis could serve the purpose of extending the sensitivity of the chromatic system in the wavelength or in the comb-frequency domain. Diurnal mid-water fish that show what is probably the largest visible window (from 350 to $800 \mathrm{~nm}$ ) encounter the first advantage among vertebrates. The absorption curves of their photoreceptors, based on porphyropsin, are slightly broader than those based on cone opsin. Thus, the comb frequency cut-off is expected to be slightly inferior to that of the human system. In this case tetrachromacy is of no advantage in the comb frequency domain (Bowmaker, 1983). 
On the other hand, in birds (Maier and Bowmaker, 1993), reptiles (Lipetz, 1984) and crustaceans (Cronin and Marshall, 1994), the spectral sensitivity curve of the pigment is narrowed by means of colored oil droplets that act as cut-off filters in the wavelength domain. The Fourier transform of a $569 \mathrm{~nm}$ photopigment with a red oil droplet for chicken retinas extends to comb frequencies nearly twice as high as that of human cones (Barlow, 1982; Bowmaker, 1983) (figure 7). The combination of oil droplet-receptor as a functional unit with a narrow spectral sensitivity curve suggests that color signals with higher band-limits exist in the environment of these species.

Under daylight illuminants, such color signals may be found among a second type of biological coloration called 'schemochrome' or structural colors. Structural colors result from the selective reflection of light by scattering, interference or diffraction produced by the interaction of the light with the microstructure of the biological material. In particular, interference colors, which are rare in plants, are widespread in the animal kingdom (wings of insects, fish scales, eye, outer

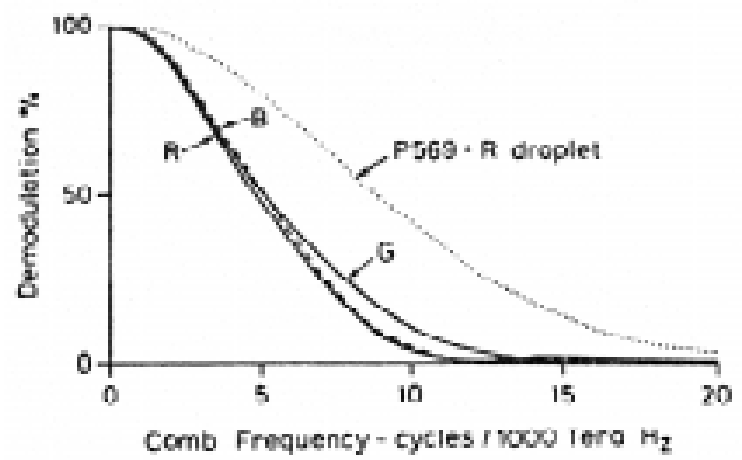

From H. Barlow, 1982

Figure 7

Compared to the Fourier transform of the three types of human photoreceptors (continuous and dashed lines), the Fourier transform of the spectral sensitivity of a chicken cone screened by an oil droplet (dotted line) indicates an extension to higher comb frequencies. It should be noted that the scale is in $\mathrm{c}$ / Tera $\mathrm{Hz}$ (each division corresponds to $1.6 \mathrm{c} / \mathrm{T}$ ), for which the responses of the three type of human receptors become similar. layer of the skin of some snakes and feathers of birds). A representative example is given by the iridescent (coherent scattering) green reflection spectra of Indian peafowl with two sharp reflectance peaks in the green region $(500 \mathrm{~nm})$ and the blue region (400 $\mathrm{nm})$ or shifted in the ultra violet range (350 $\mathrm{nm})$. The conditions are the same for the feathers of the quetzal bird (Finger and Burkhardt, 1994). A recent analysis performed by a team of ornithologists on the non-iridescent blue color (which, until now was incorrectly attributed to the Rayleigh scattering) has identified the process by which its coloration occurs. Interestingly, the predicted spectrum of this structural non-iridescent blue color also shows sharp peaks (Prum et al. 1999).

The Molecular Interaction Hypothesis does not apply to schemochrome colors and no $\mathrm{PC}$ or Fourier analysis is available to test the Low-pass hypothesis. However, considering the profile of their spectra, it is likely that structural colors encompass higher comb frequencies and that a higher number of basis functions would be required for their approximation by a linear model. As an illustration, Figure 8 presents the profile of color signals resulting from a biochrome (orange) and a schemochrome (blue feather) color under daylight or tungsten illumination. A comparison of their relative Fourier powers indicates a substantial power beyond the second Fourier component in the case of the schemochrome color, whereas it is only residual in the case of the biochrome color under both illuminants. The criteria of 99\% of total energy is reached for frequencies below $2 \mathrm{c} / \mathrm{T}$ for color signals resulting from the orange under the two illuminants, and at frequencies below $3 \mathrm{c}$ and $4 \mathrm{c} / \mathrm{T}$, for the blue feather illuminated by daylight and tungsten bulb, respectively (see Table I for values).

If the combination oil droplet-retinal receptor in some tetrachromatic species allows higher comb frequencies to pass, this introduces the possibility that the band-pass response of their chromatic systems would consequently be widened to higher comb frequencies. In this case, a new coherence would be obtained by increasing the number of color channels, narrowing the retinal 


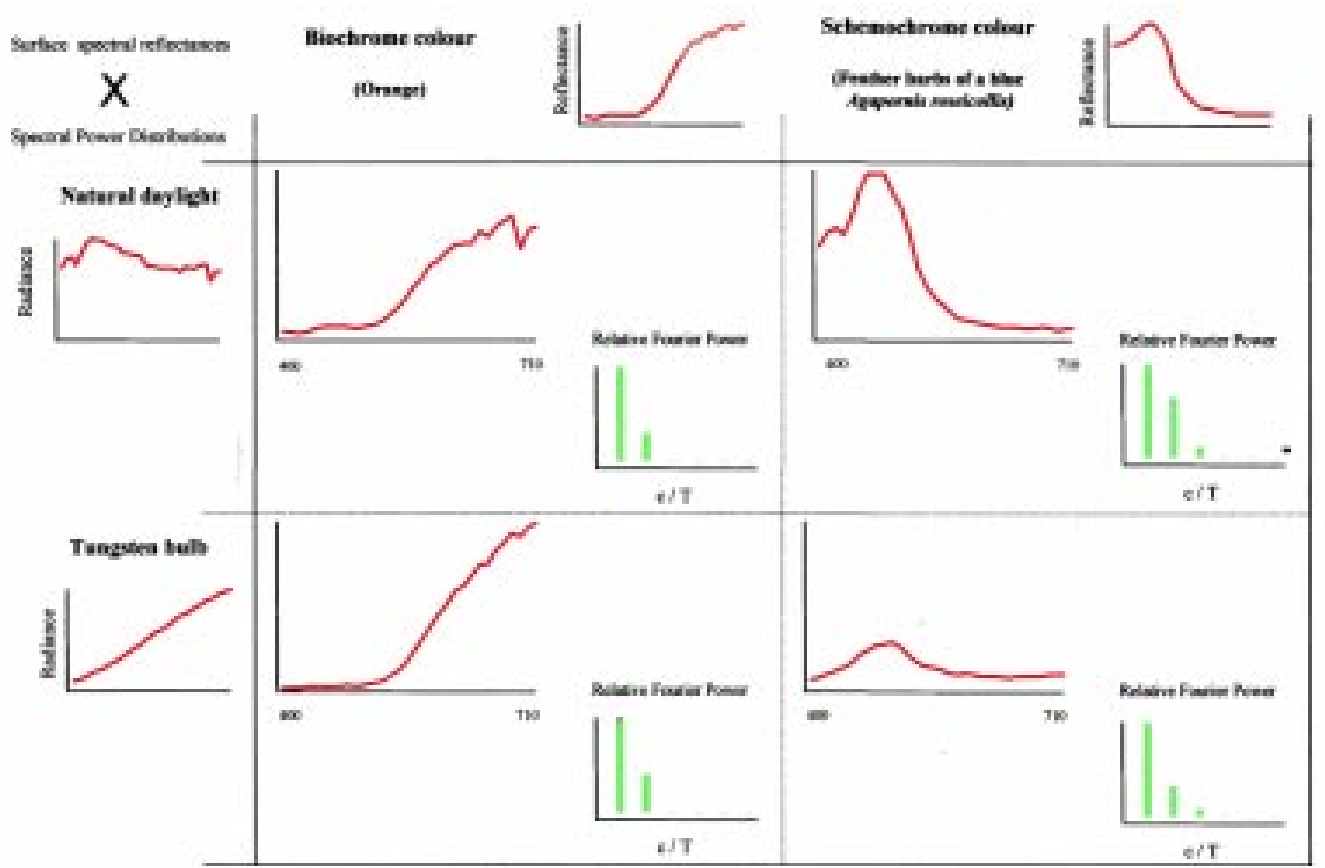

\section{Figure 8}

Four color signals and their relative Fourier Power (RFP) resulting from the product of two surface reflectances (SS) (biochrome and schemochrome colors, top row) and two illuminants (natural daylight and tungsten bulb, left column). The spectral power distribution (SPD) of the daylight illuminant is taken from Romero et al. (1997) measurements, the reflectance function of the biochrome colors from Chittka (1996) and that of the schemocrome color was reconstituted from Prum et al. (1999).

photopigments by oil droplets, and adjusting the neural mechanisms in a tetravalent manner to handle color constancy for schemochrome colors. The recent findings of Ventura, Zana, de Souza and DeVoe (Ventura et al 2001) support this hypothesis. For the first time, the authors reported a tetraphasic opponent color ganglion cell $[(\mathrm{UV}+\mathrm{M})-(\mathrm{S}+\mathrm{L})]$ in the turtle retina (Pseudemys Scripta Elegans), giving rise to the possibility that higher comb-frequency components can be processed beyond the photoreceptoral stage. However, the determination of the transfer characteristics of tetrachromatic systems, as well as the test of the Low-pass hypothesis for surface reflectances from the corresponding natural environment, are key steps towards validating the present hypothesis.

\section{REFERENCES}

BARLOW HB (1982) What causes trichromacy? A theoretical analysis using comb-filtered spectra. Vis Res 22: 635-644
BARLOW HB, GEMPERLEIN R, PAUL R, STEINER A (1983) Human contrast sensitivity for comb-filtered spectra. J Physiol, London. p:50

BONNARDEL V (1998) Demonstration of a new stimulator for human color vision testing. J Physiol, London. p:506

BONNARDEL V, VARELA F (1991) A frequency view of color: measuring the human sensitivity to squarewave spectral power distributions. Proc Roy Soc London B, 245:165-171

BONNARDEL V, BELLEMARE H, MOLLON JD (1996) Measurements of human sensitivity to comb-filtered spectra. Vis Res 36: 2713 - 2720

BONNARDEL V, RUDERMAN DL, BARLOW HB (1997) A fast determination of the spectral modulation sensitivity function : A comparison between trichromats and deuteranopes. In CAVONIUS CR (ed) Color Vision Deficiencies XIII: 415-424.

BONNARDEL V, MALONEY LT (2000) Daylight, biochrome surfaces, and human chromatic response in the Fourier domain. J Opt Soc Am A 17: 677- 686

BOWMAKER JK (1983) Trichromatic color vision: why only three channels? Trends in Neurosci 6: 41-43

BRACEWELL R (1965) The Fourier transform and its application. New York: McGraw-Hill.

BUCHSBAUM G and GOTTSCHALK A (1983) Trichromacy, opponent color coding and optimum color information transmission in the retina. Proc Roy Soc London B 220: 83-113

CHIAO C, CRONIN TW, OSORIO D (2000) Color signals in natural scenes: characteristics of reflectance spectra and effects of natural illuminants. J Opt Soc Am A $17: 218-223$ 
CHITTKA L (1996) Optimal sets of color receptors and color opponent systems for coding of natural objects insect vision. J Theo Biol 181: 179-196

COHEN J (1964) Dependency of the spectral reflectance curves of Munsell color chips. Psy Sci 1: 369- 370

COLlins M (1925) Color Blindness. Keegan Paul: London

CONWAY B (2000) Spatial structure of cone inputs to cortical color cells in alert macaque V1. Color Vision : Variance and Constancy (Conference). Newcastle: The Color Group, September 2000

CRONIN TW, MARSHALL NJ (1994) The unique visual system of the mantis shrimp. Am Sci. 82: 356-365

CRUZ-COKE R (1964) Color blindness - an evolutionary approach. Charles Thomas Springfield, IL, USA.

DERRINGTON AM, KRAUSKOPF J, LENNIE P (1984) Chromatic mechanisms in lateral geniculate nucleus of macaque. J Physiol, London. 357: 41-265

DOMINY NJ, LUCAS PW (2001) Ecological importance of trichromatic vision to primates. Nature 410:363365

FINGER E, BURKHARDT D (1994) Biological aspects of bird coloration and avian color-vision including ultraviolet range. Vis Res 34:1509-1514

GOLDSMITH TH (1991) The evolution of visual pigments and color vision. In CRONLY-DILLON J (ed) Vision and visual dysfunction. The perception of color. London: The Macmillan Press Ltd

HERING E (1878) Zur Lehre vom Lichtsinne. Wien: Carl Gerlod's Sohn

HURLBERT A (1998) Computational models of color constancy. In: WALSH V, KULIKOWSKY J (eds). Perceptual constancies. Cambridge: Cambridge University Press

HURVICH LM, JAMESON D (1957) An opponent-process theory of color vision. Psy Rev 64: 384-403

INGLING CR, TSOU HUONG-PENG B (1977) Orthogonal combination of the three visual channels. Vis Res 17: 1075-1082

JUDD DB, MACADAM DL, WYSZECKI GW (1964) Spectral distribution of typical daylight as function of correlated color temperature. J Opt Soc Am 54 :1031-1040

KRINOV EL (1947) Spectral reflectance properties of natural formations. Ottawa: National Research Council of Canada. Technical translation: TT-439

LANDAU H, POLLAK H (1962) Prolate spheroidal wave functions, Fourier analysis and uncertainty (iii): the dimension of the space of essentially time and band limited signals. Bell Sys Tech J 41:1295-1336

LIPETZ LE (1984) Pigments types, densities and concentrations in cone oil droplets of Emydoidea Blandingii. Vis Res 24:605-612

MAIER EJ, BOWMAKER JK (1993) Color vision in the passeriform bird, Leiothrix lutea: correlation of visual absorbance and oil droplet transmission with spectral sensitivity. J Comp Physiol A 172: 295-301

MALONEY LT (1986) Evaluation of linear models of surface spectral reflectance with small numbers of parameters. J Opt Soc Am A 3:1673-1681

MALONEY LT (1999) Physics-based models of surface color perception. In: GEGENFURTNER KR, SHARPE LT (eds) Color vision: from genes to perception. Cambridge: Cambridge University Press
NATHANS J, PIATANIDA TP, EDDY RL, SHOWS TB, HOGNESS DS (1986) Molecular genetics of inherited variations in human color vision. Science 232:203-10

NATHANS J, THOMAS D, HOGNESS DS (1986) Molecular genetics of human color vision: the genes encoding blue, green and red pigments. Science 232:193-202

NASSAU K (1983) The physics and chemistry of color: the fifteen causes of color. New York: Wiley

NEUMEYER C (1998) Comparative aspects of color constancy. In: WALSH V, KULIKOWSKY J, (eds). Perceptual constancies. Cambridge: Cambridge University Press

NEWTON I (1730) Opticks: or a Treatise of the Reflections, Refractions, Inflections and Colours of Lights. $4^{\text {th }}$ Edition, London: William Innys

PARKKINEN JPS, HALLOKAINEN J, JAAKELAINEN T (1989) Characteristic spectra of Munsell colors. J Opt Soc Am A 6: 318-322

PRUM RO, TORRES RH, WILLIAMSON S, DYCKJ (1999) Two-dimensional Fourier analysis of the spongy medullary keratin of structurally colored feather barbs. Proc Roy Soc London B 266: 13 - 22

REGAN BC, JULLIOT C, SIMMEN B, VIÉNOT F, CHARLES-DOMINIQUE P, MOLLON JD (1998) Frugivory and color vision in Alouatta seniculus, a trichromatic plathyrrhine monkey. Vis Res 38: 3321 3329

ROMERO J, GARCIA JA, VALERO EM, NIEVES JL (1997) Measurements of the spectral modulation sensitivity function for two normal observers with CRT monitors. J Opt Paris 28: 190-198

ROMERO J, GARCIA-BELTRAN A, HERNANDEZANDRES J (1997) Linear bases for representation of natural and artificial illuminants. J Opt Soc Am A 14: 1007-1014

RUTTIGER L, MAYSER H, SÉVEY L, SHARPE LT (2001) The color constancy of the red-green color blind. Col Res App 26: S209-S213

SHAFER SA (1985) Using color to separate reflection components. Col Res App 10: 210-218

SMITH VC, POKORNY J (1975) Spectral sensitivity of the foveal cone photopignments between 400 and 500 nm. Vis Res 15: $161-171$

STILES WS, WYSZECKI G, OHTA N. (1977) Counting metameric object-color stimuli using frequencylimited spectral reflectance functions. J Opt Soc Am 67: 779-784

THOMPSON E, PALACIOS A, VARELA F (1992). Way of coloring - Comparative color vision as a case study for cognitive science. BBS 15: 1-74

THOMPSON E (1995) Color Vision. London: Routledge Kegan Paul

VAN HATEREN JH (1993) Spatial, temporal and spectral pre-processing for color vision. Proc Roy Soc London 251: 61-68

VENTURA DF, ZANA Y, DE SOUZA JM, DEVOE RD (2001) UV color opponency in the turtle retina. J Exp Biol 204: 2527-2534

VRHEL MJ, GERSHON R, IWAN LS (1994) Measurement and analysis of object reflectance spectra. Col Res App 19:4 - 9

YOUNG T (1802) The Bakerian lecture : On the theory of light and colors. Phil Trans Roy Soc London 92:12 - 48 
APPENDIX A

A real-valued function cannot be localized in the variable (say within an interval $\mathrm{T}$ ) and in the frequency domain (say in an interval B) at the same time. The best that can be achieved is a compromise such as that of Gaussian profiles of intermediate spread. This 'limitation' result for bandlimited functions is well known and takes different forms, the most famous being the Heisenberg uncertainty principle. It is also the basis of what is known as the 'folk' version of the so-called Sampling Theorem. Roughly stated, the Sampling Theorem asserts that one can recover (up to negligible error) a band limited signal from $2 \mathrm{BT}$ sampling or basis functions. In this form the folk Sampling Theorem is usually attributed to Whittaker and Shannon, who first used it in the 1940's in an empirical fashion. A rigorous justification was not available until Landau and Pollak (1962), motivated by the use of time and band limited functions in communication theory, achieved their result through a very extensive study of time and band limited functions. Their results apply to the ensemble of square-integrable real functions, which are said to be approximately variable-limited to $|x| \leq T / 2$, that is:

$$
\frac{\left.|| f(x)\right|^{2} d x}{\int_{-\infty}^{\infty x \mid \leq T / 2}|f(x)|^{2} d x}=1-\varepsilon_{T}^{2}
$$

where one assumes that $\varepsilon_{T}^{2}$ is small and, under normalization of the unit area, measures the degree to which $f(x)$ fails to be concentrated in the interval $|x| \leq T / 2$. Similarly, one assumes that the Fourier transform $f(\omega)$ of a function $f(x)$ nearly vanishes for $|\omega| \leq 2 \pi B$. Under these conditions, Landau and Pollak (1962) show there is a linear model based on $2 \mathrm{BT}$ independent functions that approximate any function with a frequency limit given by B, with an error comparable to $\varepsilon_{T}^{2}$. This linear model is defined by wavelets described by the so-called 'prolate spheroidal functions,', which are the optimal base for this type of functions. Any other orthonormal set is not as efficient, in that it can be shown that the number of required functions grows beyond 2BT quite rapidly. We call this a strict relationship between band limitation and sampling basis. 